\title{
Ad Hoc Reviewer Acknowledgments
}

The International Journal of Stress Management depends on the goodwill of many experts who help review manuscripts that have been submitted for publication. In addition to the Editorial Board members, the following individuals reviewed manuscripts that were considered for the 2018 volume. The editor acknowledges all reviewers and thanks them for their time and effort.

Saima Ahmad

Toni Alterman

Alberto Amutio-Kareaga

Hossein Analuie

Paul T. Aquino

Sophie Baeriswyl

Anja Baethge

Colter Bayard

Randal D. Beaton

Tracy A. Beegen

Kristen Jennings Black

Petri Bockerman

Carolyn M. Boyd

David L. Breen

Daniel M. Campagne

David Caruso

Anne Casper

Maria José Chambel

Roxane Chan

Francis Cheung

Andrew Clements

Keren Cohen-Louck

Lesley Collier

Shengli Dong

Luis Manuel Blanco

Donoso

Joana Duarte

Joanna Dziura

Kevin Eschleman

Andrea Fischbach

Antonio Font

Anne Marie Francesco
Markus Gerber

Bradley L. Graham

Paula Grubb

Mike Hammes

Joseph Neil Henderson

Kristin Horan

Victoria Hui

Shuhei Iimura

Paulino Jimenez

Robert Juster

David Kealy

Anne-Kathrin Konze

Jean Kristeller

Bettina Kubicek

Julian C. L. Lai

Bonnie Lee

Elizabeth Lee

Stephen Levine

Yiqiong $\mathrm{Li}$

Jiafang Lu

Vivian Lun

Jie Ma

Daniel Ruivo Marques

Viviane Masciotra*

Liz Munoz

Amy Murrell

Julian Mutz

Karen Niven

Gina P. Owens
Grete Patil

Shani Pindek

Thomas G. Plante

Roman Prem

Alessandro Lo Presti

Jane Price

Thomas Rigotti

Wladislaw Rivkin

Lara Christina Roll

Simon Rosenbaum

Gargi Sawhney

April D. Schantz

Ben Searle

Ana Junça Silva

Wesley Sime

Jacob Stein

Dhriti Tiwari*

Dana Unger

Biljana van Rijn

Oliver Weigelt

Dana Yagil

Tianan Yang

Jan Fekke Ybema

Chao Ye

Moshe Zeidner

Ran Zhang

Xichao Zhang

Hao Zhao

Clare Zhao*

Biru Zhou

* Denotes co-reviewers who reviewed under the supervision of a primary reviewer. 